\title{
Third-order integrable difference equations generated by a pair of second-order equations
}

\author{
Junta Matsukidaira* \\ Department of Applied Mathematics and Informatics, \\ Ryukoku University, Seta, Otsu, Shiga 520-2194, Japan \\ Daisuke Takahash \\ Department of Mathematical Sciences, \\ Waseda University, 3-4-1, Ohkubo, Shinjuku-ku, Tokyo 169-8555, Japan
}

(Dated: October 16, 2018)

\begin{abstract}
We show that the third-order difference equations proposed by Hirota, Kimura and Yahagi are generated by a pair of second-order difference equations. In some cases, the pair of the second-order equations are equivalent to the Quispel-Robert-Thomson(QRT) system, but in the other cases, they are irrelevant to the QRT system. We also discuss an ultradiscretization of the equations.
\end{abstract}

PACS numbers: 02.30Ik, 05.45.-a

*Electronic address: junta@math.ryukoku.ac.jp

${ }^{\dagger}$ Electronic address: daisuket@waseda.jp 


\section{INTRODUCTION}

Discrete integrable systems have attracted much attention and a lot of studies have been done by various points of view, such as integrability criteria (singularity confinement property 1], algebraic entropy [2]), geometric or algebraic description of the equations [3, 4, [5, 6, 7, 8] and so on.

Especially, second-order integrable difference equations including QRT system [9, 10] and discrete Painlevé equations[11] which are regarded as non-autonomous variations of QRT system, have been extensively studied, and a number of significant properties have been obtained.

For example, a symmetric version of QRT system is defined by the following form

$$
x_{n+1}=\frac{f_{1}\left(x_{n}\right)-x_{n-1} f_{2}\left(x_{n}\right)}{f_{2}\left(x_{n}\right)-x_{n-1} f_{3}\left(x_{n}\right)},
$$

where $f_{j}(x)$ is defined by

$$
\left(\begin{array}{l}
f_{1}(x) \\
f_{2}(x) \\
f_{3}(x)
\end{array}\right)=A\left(\begin{array}{c}
x^{2} \\
x \\
1
\end{array}\right) \times B\left(\begin{array}{l}
x^{2} \\
x \\
1
\end{array}\right)
$$

with arbitrary symmetric $3 \times 3$ matrices $A$ and $B$. This equation has a conserved quantity $h\left(x_{n-1}, x_{n}\right)$ defined by $A$ and $B$. Moreover, a general solution is described by an elliptic function.

However very few results have been obtained for third-order integrable difference equations. Research on such equations are important in order to reveal integrable structures of general discrete integrable systems.

In this paper, we investigate third-order integrable difference equations proposed by Hirota, Kimura and Yahagi[12] and show that they are generated by a pair of second-order integrable difference equations. Moreover we also discuss their ultradiscretization.

Hirota, Kimura and Yahagi have investigated third order difference equations of the form

$$
x_{n+2} x_{n-1}=\frac{a_{0}+a_{1} x_{n}+a_{2} x_{n+1}+a_{3} x_{n} x_{n+1}}{b_{0}+b_{1} x_{n}+b_{2} x_{n+1}+b_{3} x_{n} x_{n+1}},
$$


and have found that nine equations,

$$
\begin{aligned}
& x_{n+2} x_{n-1}=\frac{a_{0}+a_{1}\left(x_{n}+x_{n+1}\right)+a_{3} x_{n} x_{n+1}}{a_{3}+b_{1}\left(x_{n}+x_{n+1}\right)+b_{3} x_{n} x_{n+1}}, \\
& x_{n+2} x_{n-1}=\frac{a_{0}\left(1+x_{n}+x_{n+1}\right)+a_{3} x_{n} x_{n+1}}{a_{0}+a_{3}\left(x_{n}+x_{n+1}+x_{n} x_{n+1}\right)}, \\
& x_{n+2} x_{n-1}=\frac{a_{0}\left(-1+x_{n}-x_{n+1}\right)+a_{3} x_{n} x_{n+1}}{a_{0}+a_{3}\left(x_{n}-x_{n+1}-x_{n} x_{n+1}\right)}, \\
& x_{n+2} x_{n-1}=\frac{a_{0}+a_{1}\left(x_{n}+x_{n+1}+x_{n} x_{n+1}\right)}{1+x_{n}+x_{n+1}+x_{n} x_{n+1}}, \\
& x_{n+2} x_{n-1}=\frac{a_{1}\left(x_{n}-x_{n+1}\right)+a_{3} x_{n} x_{n+1}}{a_{3}+b_{1}\left(-x_{n}+x_{n+1}\right)}, \\
& x_{n+2} x_{n-1}=\frac{a_{3} x_{n} x_{n+1}}{b_{1}\left(x_{n}+x_{n+1}\right)+b_{3} x_{n} x_{n+1}}, \\
& x_{n+2} x_{n-1}=\frac{a_{0}+a_{1} x_{n}}{a_{1} x_{n}+a_{0} x_{n} x_{n+1}}, \\
& x_{n+2} x_{n-1}=\frac{a_{0}+a_{1} x_{n}}{-a_{1} x_{n}+a_{0} x_{n} x_{n+1}}, \\
& x_{n+2} x_{n-1}=\frac{x_{n}+x_{n} x_{n+1}}{1+x_{n}},
\end{aligned}
$$

are integrable in the sense that they have two independent conserved quantities. A remarkable property of these equations is that their trajectory of a solution in 3-dimensional phase space looks like a composition of two separate curves. Fig. 1 is an example of such trajectories in $3 \mathrm{D}$ phase space which is generated by

$$
y_{n+2} y_{n-1}=a+y_{n}+y_{n+1},
$$

where the equation is obtained through a variable transformation $y_{n}=\frac{a_{3}}{b_{1} x_{n}}, a=\frac{a_{3} b_{3}}{b_{1}^{2}}$ from Eq. (Y6). Moreover, it is an important fact that odd step points belong to the one curve and even step points belong to the other.

This fact strongly suggests that a combination of lower dimensional integrable equations determines the integrability of the third-order difference equation. We show this is true for all nine equations in the following section. 


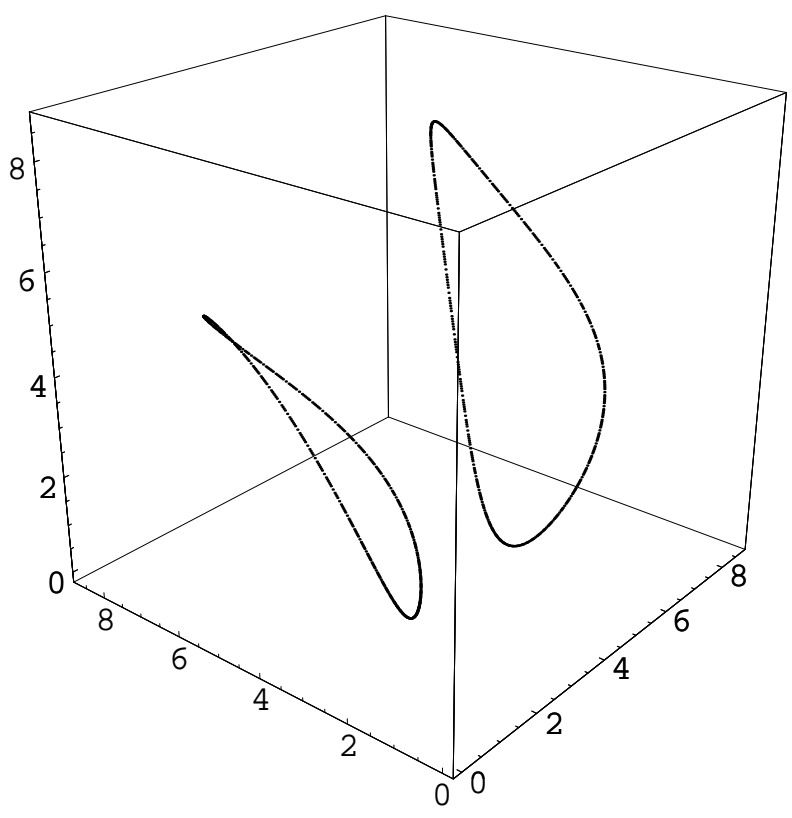

FIG. 1: A trajectory of a solution to Eq. (4) for $a=2.0, y_{0}=1.0, y_{1}=2.0, y_{2}=1.5$

\section{PAIR OF SECOND-ORDER INTEGRABLE EQUATIONS GENERATING A THIRD-ORDER EQUATION}

\section{A. Y6}

If we take a backward difference of Eq. (4)

$$
y_{n+2} y_{n-1}=a+y_{n}+y_{n+1}
$$

we obtain

$$
\Delta_{n}\left(y_{n+2} y_{n-1}-a-y_{n}-y_{n+1}\right)=0
$$

where $\Delta_{n}$ is a difference operator defined by $\Delta_{n} f_{n}=f_{n}-f_{n-1}$. Eq. (15) can be written as

$$
\frac{\left(1+y_{n+2}\right)\left(1+y_{n}\right)}{y_{n+1}}=\frac{\left(1+y_{n}\right)\left(1+y_{n-2}\right)}{y_{n-1}} \text {. }
$$

This formula means that there are constants which depend on the initial values and on a parity of $n$. Hence, we obtain

$$
\left\{\begin{array}{l}
\frac{\left(1+g_{n+1}\right)\left(1+g_{n}\right)}{h_{n}}=c_{0}, \\
\frac{\left(1+h_{n}\right)\left(1+h_{n-1}\right)}{g_{n}}=c_{1},
\end{array}\right.
$$


where $g_{n}=y_{2 n}, \quad h_{n}=y_{2 n+1}, \quad c_{0}=\frac{\left(1+y_{0}\right)\left(1+y_{2}\right)}{y_{1}}, \quad c_{1}=\frac{\left(1+y_{1}\right)\left(1+y_{3}\right)}{y_{2}}$. From Eqs. (41) and (7), we obtain a pair of second-order difference equations

$$
\begin{aligned}
g_{n+1} & =\frac{\left(1+a c_{0}\right)+\left(1+c_{0}\right) g_{n}}{g_{n-1}\left(1+g_{n}\right)}, \\
h_{n+1} & =\frac{\left(1+a c_{1}\right)+\left(1+c_{1}\right) h_{n}}{h_{n-1}\left(1+h_{n}\right)},
\end{aligned}
$$

where Eq. (8) is a equation for even steps and Eq. (99) is a equation for odd steps respectively. Eq. (8) and Eq. (91) can be written in the QRT form

$$
\left\{\begin{array}{l}
g_{n+1}=\frac{G_{1}\left(g_{n}\right)-g_{n-1} G_{2}\left(g_{n}\right)}{G_{2}\left(g_{n}\right)-g_{n-1} G_{3}\left(g_{n}\right)} \\
h_{n+1}=\frac{H_{1}\left(h_{n}\right)-h_{n-1} H_{2}\left(h_{n}\right)}{H_{2}\left(h_{n}\right)-h_{n-1} H_{3}\left(h_{n}\right)},
\end{array}\right.
$$

where

$$
\begin{aligned}
A(c) & =\left(\begin{array}{ccc}
1 & 2+c & 1+c \\
2+c & 0 & 2+2 c+a c+c^{2} \\
1+c & 2+2 c+a c+c^{2} & (1+c)(1+a c)
\end{array}\right), \quad B=\left(\begin{array}{lll}
0 & 0 & 0 \\
0 & 1 & 0 \\
0 & 0 & 0
\end{array}\right), \\
\left(\begin{array}{l}
G_{1}(x) \\
G_{2}(x) \\
G_{3}(x)
\end{array}\right) & =A\left(c_{0}\right)\left(\begin{array}{l}
x^{2} \\
x \\
1
\end{array}\right) \times B\left(\begin{array}{l}
x^{2} \\
x \\
1
\end{array}\right), \quad\left(\begin{array}{l}
H_{1}(x) \\
H_{2}(x) \\
H_{3}(x)
\end{array}\right)=A\left(c_{1}\right)\left(\begin{array}{l}
x^{2} \\
x \\
1
\end{array}\right) \times B\left(\begin{array}{l}
x^{2} \\
x \\
1
\end{array}\right) .
\end{aligned}
$$

Consequently, conserved quantities of Eqs. (8) and (91) are given as

$$
\begin{aligned}
k_{0}= & \left(g_{n}^{2} g_{n+1}^{2}+\left(2+c_{0}\right)\left(g_{n}+g_{n+1}\right) g_{n} g_{n+1}+\left(1+c_{0}\right)\left(g_{n}^{2}+g_{n+1}^{2}\right)\right. \\
& \left.+\left(2+2 c_{0}+a c_{0}+c_{0}^{2}\right)\left(g_{n}+g_{n+1}\right)+\left(1+c_{0}\right)\left(1+a c_{0}\right)\right) /\left(g_{n} g_{n+1}\right),
\end{aligned}
$$

and

$$
\begin{aligned}
k_{1}= & \left(h_{n}^{2} h_{n+1}^{2}+\left(2+c_{1}\right)\left(h_{n}+h_{n+1}\right) h_{n} h_{n+1}+\left(1+c_{1}\right)\left(h_{n}^{2}+h_{n+1}^{2}\right)\right. \\
& \left.+\left(2+2 c_{1}+a c_{1}+c_{1}^{2}\right)\left(h_{n}+h_{n+1}\right)+\left(1+c_{1}\right)\left(1+a c_{1}\right)\right) /\left(h_{n} h_{n+1}\right) .
\end{aligned}
$$

Hence invariant curves of Eqs. (8) and (91) are given by the above equations. These curves determine the structure of the trajectory of a solution to Eq. (Y6) in 3D phase space shown in Fig. 1. This is the simplified integrability structure of Eq. (Y6) and we show below a similar structure exists in the other eight equations. 


\section{B. $\mathrm{Y} 1$}

From Eq. (Y1)

$$
x_{n+2} x_{n-1}=\frac{a_{0}+a_{1}\left(x_{n}+x_{n+1}\right)+a_{3} x_{n} x_{n+1}}{a_{3}+b_{1}\left(x_{n}+x_{n+1}\right)+b_{3} x_{n} x_{n+1}}
$$

we obtain

$$
\Delta_{n}\left(\left(a_{3}+b_{1}\left(x_{n}+x_{n+1}\right)+b_{3} x_{n} x_{n+1}\right) x_{n+2} x_{n-1}-\left(a_{0}+a_{1}\left(x_{n}+x_{n+1}\right)+a_{3} x_{n} x_{n+1}\right)\right)=0 .
$$

Eq. (15) can be written as

$$
\begin{aligned}
& b_{3} x_{n+2} x_{n}+b_{1}\left(x_{n+2}+x_{n}+\frac{x_{n+2} x_{n}}{x_{n+1}}\right)+a_{3}\left(\frac{x_{n+2}}{x_{n+1}}+\frac{x_{n}}{x_{n+1}}\right)+\frac{a_{1}}{x_{n+1}} \\
& =b_{3} x_{n} x_{n-2}+b_{1}\left(x_{n}+x_{n-2}+\frac{x_{n} x_{n-2}}{x_{n-1}}\right)+a_{3}\left(\frac{x_{n}}{x_{n-1}}+\frac{x_{n-2}}{x_{n-1}}\right)+\frac{a_{1}}{x_{n-1}} .
\end{aligned}
$$

Hence we obtain

$$
\left\{\begin{array}{l}
g_{n}=\frac{a_{1}+a_{3}\left(h_{n-1}+h_{n}\right)+b_{1} h_{n-1} h_{n}}{c_{1}-b_{1}\left(h_{n-1}+h_{n}\right)-b_{3} h_{n-1} h_{n}}, \\
h_{n}=\frac{a_{1}+a_{3}\left(g_{n}+g_{n+1}\right)+b_{1} g_{n} g_{n+1}}{c_{0}-b_{1}\left(g_{n}+g_{n+1}\right)-b_{3} g_{n} g_{n+1}},
\end{array}\right.
$$

where $g_{n}=x_{2 n}, h_{n}=x_{2 n+1}$ and

$$
\begin{aligned}
& c_{0}=\frac{1}{x_{1}}\left(b_{1} x_{0} x_{2}+a_{3} x_{2}+a_{3} x_{0}+a_{1}\right)+b_{3} x_{0} x_{2}+b_{1}\left(x_{0}+x_{2}\right), \\
& c_{1}=\frac{1}{x_{2}}\left(b_{1} x_{1} x_{3}+a_{3} x_{3}+a_{3} x_{1}+a_{1}\right)+b_{3} x_{1} x_{3}+b_{1}\left(x_{1}+x_{3}\right) .
\end{aligned}
$$

From Eqs. (Y1) and (17), we obtain a pair of QRT systems

$$
\left\{\begin{array}{l}
g_{n+1}=\frac{G_{1}\left(g_{n}\right)-g_{n-1} G_{2}\left(g_{n}\right)}{G_{2}\left(g_{n}\right)-g_{n-1} G_{3}\left(g_{n}\right)} \\
h_{n+1}=\frac{H_{1}\left(h_{n}\right)-h_{n-1} H_{2}\left(h_{n}\right)}{H_{2}\left(h_{n}\right)-h_{n-1} H_{3}\left(h_{n}\right)},
\end{array}\right.
$$

where

$$
\begin{aligned}
& A(c)=\left(\begin{array}{ccc}
b_{1}^{2}-2 a_{3} b_{3}+\frac{a_{1} b_{3}^{2}}{b_{1}} & 0 & a_{3}^{2}-a_{1} b_{1} \\
0 & 2 a_{3}^{2}-a_{0} b_{3}+2 a_{3} c-\frac{a_{1} b_{3} c}{b_{1}} & 2 a_{1} a_{3}-a_{0} b_{1}+a_{1} c \\
a_{3}^{2}-a_{1} b_{1} & 2 a_{1} a_{3}-a_{0} b_{1}+a_{1} c & a_{1}^{2}+a_{0} c
\end{array}\right), \\
& B(c)=\left(\begin{array}{ccc}
b_{3} & b_{1} & 0 \\
b_{1} & -c & 0 \\
0 & 0 & 0
\end{array}\right)
\end{aligned}
$$




$$
\left(\begin{array}{l}
G_{1}(x) \\
G_{2}(x) \\
G_{3}(x)
\end{array}\right)=A\left(c_{0}\right)\left(\begin{array}{l}
x^{2} \\
x \\
1
\end{array}\right) \times B\left(c_{0}\right)\left(\begin{array}{c}
x^{2} \\
x \\
1
\end{array}\right), \quad\left(\begin{array}{l}
H_{1}(x) \\
H_{2}(x) \\
H_{3}(x)
\end{array}\right)=A\left(c_{1}\right)\left(\begin{array}{c}
x^{2} \\
x \\
1
\end{array}\right) \times B\left(c_{1}\right)\left(\begin{array}{c}
x^{2} \\
x \\
1
\end{array}\right)
$$

C. $\mathrm{Y} 4$

From Eq. (Y4)

$$
x_{n+2} x_{n-1}=\frac{a_{0}+a_{1}\left(x_{n+1}+x_{n}+x_{n+1} x_{n}\right)}{1+x_{n}+x_{n+1}+x_{n+1} x_{n}},
$$

we obtain

$$
\Delta_{n}\left(\left(1+x_{n}+x_{n+1}+x_{n+1} x_{n}\right) x_{n+2} x_{n-1}-\left(a_{0}+a_{1}\left(x_{n+1}+x_{n}+x_{n+1} x_{n}\right)\right)\right)=0 .
$$

Eq. (23) can be written as

$$
\left(x_{n+2}+a_{1}\right)\left(x_{n}+a_{1}\right) \frac{x_{n+1}+1}{x_{n+1}}=\left(x_{n}+a_{1}\right)\left(x_{n-2}+a_{1}\right) \frac{x_{n-1}+1}{x_{n-1}} .
$$

From this equation, we obtain

$$
\left\{\begin{array}{l}
g_{n}=\frac{\left(h_{n}+a_{1}\right)\left(h_{n-1}+a_{1}\right)}{c_{1}-\left(h_{n}+a_{1}\right)\left(h_{n-1}+a_{1}\right)} \\
h_{n}=\frac{\left(g_{n+1}+a_{1}\right)\left(g_{n}+a_{1}\right)}{c_{0}-\left(g_{n+1}+a_{1}\right)\left(g_{n}+a_{1}\right)}
\end{array}\right.
$$

where $g_{n}=x_{2 n}, h_{n}=x_{2 n+1}$ and

$$
\begin{aligned}
& c_{0}=\left(x_{2}+a_{1}\right)\left(x_{0}+a_{1}\right) \frac{x_{1}+1}{x_{1}}, \\
& c_{1}=\left(x_{3}+a_{1}\right)\left(x_{1}+a_{1}\right) \frac{x_{2}+1}{x_{2}} .
\end{aligned}
$$

From Eqs. (Y4) and (25), we obtain a pair of QRT systems

$$
\left\{\begin{array}{l}
g_{n+1}=\frac{G_{1}\left(g_{n}\right)-g_{n-1} G_{2}\left(g_{n}\right)}{G_{2}\left(g_{n}\right)-g_{n-1} G_{3}\left(g_{n}\right)} \\
h_{n+1}=\frac{H_{1}\left(h_{n}\right)-h_{n-1} H_{2}\left(h_{n}\right)}{H_{2}\left(h_{n}\right)-h_{n-1} H_{3}\left(h_{n}\right)}
\end{array}\right.
$$


where

$$
\begin{aligned}
& A(c)=\left(\begin{array}{ccc}
\left(a_{0}-a_{1}-c\right)\left(a_{1}-1\right) & 0 & -\left(a_{0}-a_{1}-c\right)\left(a_{1}-1\right) a_{1}^{2} \\
0 & a_{22} & a_{23} \\
-\left(a_{0}-a_{1}-c\right)\left(a_{1}-1\right) a_{1}^{2} & a_{32} & a_{33}
\end{array}\right) \\
& a_{22}=-2\left(a_{0}-a_{1}\right)\left(a_{1}-1\right) a_{1}^{2}+c\left(a_{0}-2 a_{1}+a_{1}^{3}-c\right), \\
& a_{23}=-2\left(a_{0}-a_{1}\right)\left(a_{1}-1\right) a_{1}^{3}+c a_{1}\left(-a_{0}+2 a_{0} a_{1}-2 a_{1}^{2}+a_{1}^{3}-a_{1} c\right), \\
& a_{32}=a_{23}, \\
& a_{33}=-\left(a_{0}-a_{1}\right)\left(a_{1}-1\right) a_{1}^{4}+c a_{1}\left(-a_{0} a_{1}+2 a_{0} a_{1}^{2}-a_{1}^{3}-a_{0} c\right) \\
& B(c)=\left(\begin{array}{ccc}
1 & a_{1} & 0 \\
a_{1} & a_{1}^{2}-c & 0 \\
0 & 0 & 0
\end{array}\right) \\
& \left(\begin{array}{l}
G_{1}(x) \\
G_{2}(x) \\
G_{3}(x)
\end{array}\right)=A\left(c_{0}\right)\left(\begin{array}{c}
x^{2} \\
x \\
1
\end{array}\right) \times B\left(c_{0}\right)\left(\begin{array}{c}
x^{2} \\
x \\
1
\end{array}\right), \quad\left(\begin{array}{c}
H_{1}(x) \\
H_{2}(x) \\
H_{3}(x)
\end{array}\right)=A\left(c_{1}\right)\left(\begin{array}{l}
x^{2} \\
x \\
1
\end{array}\right) \times B\left(c_{1}\right)\left(\begin{array}{c}
x^{2} \\
x \\
1
\end{array}\right)
\end{aligned}
$$

From Eq. (Y5

$$
x_{n+2} x_{n-1}=\frac{a_{1} x_{n}-a_{1} x_{n+1}+a_{3} x_{n} x_{n+1}}{a_{3}-b_{1} x_{n}+b_{1} x_{n+1}} .
$$

we obtain

$$
\begin{aligned}
& x_{n+2} x_{n-1}\left(a_{3}-b_{1} x_{n}+b_{1} x_{n+1}\right)-\left(a_{1} x_{n}-a_{1} x_{n+1}+a_{3} x_{n} x_{n+1}\right) \\
& +x_{n+1} x_{n-2}\left(a_{3}-b_{1} x_{n-1}+b_{1} x_{n}\right)-\left(a_{1} x_{n-1}-a_{1} x_{n}+a_{3} x_{n-1} x_{n}\right)=0 .
\end{aligned}
$$

Note that we don't take a backward difference but a sum of Eq. (IY5) here. Eq. (32) can be written as

$$
a_{3} \frac{x_{n+2}-x_{n}}{x_{n+1}}-\frac{a_{1}}{x_{n+1}}+b_{1}\left(x_{n+2}+x_{n}\right)-b_{1} \frac{x_{n+2} x_{n}}{x_{n+1}}=a_{3} \frac{x_{n}-x_{n-2}}{x_{n-1}}-\frac{a_{1}}{x_{n-1}}+b_{1}\left(x_{n}+x_{n-2}\right)-b_{1} \frac{x_{n} x_{n-2}}{x_{n-1}} .
$$


From this equation, we obtain

$$
\left\{\begin{array}{l}
g_{n}=\frac{a_{3}\left(h_{n}-h_{n-1}\right)-a_{1}-b_{1} h_{n} h_{n-1}}{c_{1}-b_{1}\left(h_{n}+h_{n-1}\right)}, \\
h_{n}=\frac{a_{3}\left(g_{n+1}-g_{n}\right)-a_{1}-b_{1} g_{n+1} g_{n}}{c_{0}-b_{1}\left(g_{n+1}+g_{n}\right)},
\end{array}\right.
$$

where $g_{n}=x_{2 n}, h_{n}=x_{2 n+1}$ and

$$
\begin{aligned}
& c_{0}=a_{3} \frac{x_{2}-x_{0}}{x_{1}}-\frac{a_{1}}{x_{1}}+b_{1}\left(x_{2}+x_{0}\right)-b_{1} \frac{x_{2} x_{0}}{x_{1}}, \\
& c_{1}=a_{3} \frac{x_{3}-x_{1}}{x_{2}}-\frac{a_{1}}{x_{2}}+b_{1}\left(x_{3}+x_{1}\right)-b_{1} \frac{x_{3} x_{1}}{x_{2}} .
\end{aligned}
$$

From Eqs. (Y5) and (34), we obtain a pair of QRT systems

$$
\left\{\begin{array}{l}
g_{n+1}=\frac{G_{1}\left(g_{n}\right)-g_{n-1} G_{2}\left(g_{n}\right)}{G_{2}\left(g_{n}\right)-g_{n-1} G_{3}\left(g_{n}\right)} \\
h_{n+1}=\frac{H_{1}\left(h_{n}\right)-h_{n-1} H_{2}\left(h_{n}\right)}{H_{2}\left(h_{n}\right)-h_{n-1} H_{3}\left(h_{n}\right)}
\end{array}\right.
$$

where

$$
\begin{gathered}
A(c)=\left(\begin{array}{ccc}
b_{1}^{2} & 0 & -a_{3}^{2}-a_{1} b_{1} \\
0 & 2 a_{3}^{2} & a_{1} c \\
-a_{3}^{2}-a_{1} b_{1} & a_{1} c & a_{1}^{2}
\end{array}\right), \\
B(c)=\left(\begin{array}{ccc}
0 & b_{1} & 0 \\
b_{1} & -c & 0 \\
0 & 0 & 0
\end{array}\right), \\
\left(\begin{array}{l}
G_{1}(x) \\
G_{2}(x) \\
G_{3}(x)
\end{array}\right)=A\left(c_{0}\right)\left(\begin{array}{c}
x^{2} \\
x \\
1
\end{array}\right) \times B\left(c_{0}\right)\left(\begin{array}{c}
x^{2} \\
x \\
1
\end{array}\right),
\end{gathered}
$$

\section{E. $\quad$ Y7}

Introducing a variable transformation $x_{n}=\frac{f_{n+1}}{f_{n}}$ to Eq. (Y7)

$$
x_{n+2} x_{n-1}=\frac{a_{0}+a_{1} x_{n}}{a_{1} x_{n}+a_{0} x_{n} x_{n+1}},
$$


we obtain

$$
a_{0}\left(f_{n+1}+f_{n-1}\right)+a_{1} \frac{f_{n+1} f_{n-1}}{f_{n}}=a_{0}\left(f_{n+3}+f_{n+1}\right)+a_{1} \frac{f_{n+3} f_{n+1}}{f_{n+2}} .
$$

From this equation, we obtain

$$
\left\{\begin{array}{l}
a_{0}\left(g_{n+1}+g_{n}\right)+a_{1} \frac{g_{n+1} g_{n}}{h_{n}}=c_{0} \\
a_{0}\left(h_{n}+h_{n-1}\right)+a_{1} \frac{h_{n} h_{n-1}}{g_{n}}=c_{1}
\end{array}\right.
$$

where $g_{n}=f_{2 n}, h_{n}=f_{2 n+1}$ and

$$
\begin{aligned}
& c_{0}=a_{0}\left(g_{1}+g_{0}\right)+a_{1} \frac{g_{1} g_{0}}{h_{0}}=f_{0} a_{0}\left(1+x_{0} x_{1}+\frac{a_{1}}{a_{0}} x_{1}\right), \\
& c_{1}=a_{0}\left(h_{1}+h_{0}\right)+a_{1} \frac{h_{1} h_{0}}{g_{1}}=f_{0} a_{0} x_{0}\left(1+x_{1} x_{2}+\frac{a_{1}}{a_{0}} x_{2}\right) .
\end{aligned}
$$

From Eq. (42), we obtain a pair of QRT system

$$
\left\{\begin{array}{l}
g_{n+1}=\frac{G_{1}\left(g_{n}\right)-g_{n-1} G_{2}\left(g_{n}\right)}{G_{2}\left(g_{n}\right)-g_{n-1} G_{3}\left(g_{n}\right)} \\
h_{n+1}=\frac{H_{1}\left(h_{n}\right)-h_{n-1} H_{2}\left(h_{n}\right)}{H_{2}\left(h_{n}\right)-h_{n-1} H_{3}\left(h_{n}\right)},
\end{array}\right.
$$

where

$$
\begin{aligned}
& \left(\begin{array}{l}
G_{1}(x) \\
G_{2}(x) \\
G_{3}(x)
\end{array}\right)=\left(\begin{array}{ccc}
0 & 0 & a_{0}^{2}\left(a_{0}^{2}-a_{1}^{2}\right) \\
0 & \left(a_{0}^{2}-a_{1}^{2}\right)\left(2 a_{0}^{2}-a_{1}^{2}\right) & -a_{0}\left(2 a_{0}^{2} c_{0}-a_{1}^{2} c_{0}+a_{0} a_{1} c_{1}\right) \\
a_{0}^{2}\left(a_{0}^{2}-a_{1}^{2}\right) & -a_{0}\left(2 a_{0}^{2} c_{0}-a_{1}^{2} c_{0}+a_{0} a_{1} c_{1}\right) & a_{0} c_{0}\left(a_{0} c_{0}+a_{1} c_{1}\right)
\end{array}\right)\left(\begin{array}{l}
x^{2} \\
x \\
1
\end{array}\right) \\
& \times\left(\begin{array}{ccc}
a_{0} a_{1} & a_{0} c_{1} & 0 \\
a_{0} c_{1} & -c_{0} c_{1} & 0 \\
0 & 0 & 0
\end{array}\right)\left(\begin{array}{c}
x^{2} \\
x \\
1
\end{array}\right) \\
& \left(\begin{array}{l}
H_{1}(x) \\
H_{2}(x) \\
H_{3}(x)
\end{array}\right)=\left(\begin{array}{ccc}
0 & 0 & a_{0}^{2}\left(a_{0}^{2}-a_{1}^{2}\right) \\
0 & \left(a_{0}^{2}-a_{1}^{2}\right)\left(2 a_{0}^{2}-a_{1}^{2}\right) & -a_{0}\left(2 a_{0}^{2} c_{1}-a_{1}^{2} c_{1}+a_{0} a_{1} c_{0}\right) \\
a_{0}^{2}\left(a_{0}^{2}-a_{1}^{2}\right) & -a_{0}\left(2 a_{0}^{2} c_{1}-a_{1}^{2} c_{1}+a_{0} a_{1} c_{0}\right) & a_{0} c_{1}\left(a_{0} c_{1}+a_{1} c_{0}\right)
\end{array}\right)\left(\begin{array}{l}
x^{2} \\
x \\
1
\end{array}\right) \\
& \times\left(\begin{array}{ccc}
a_{0} a_{1} & a_{0} c_{0} & 0 \\
a_{0} c_{0} & -c_{0} c_{1} & 0 \\
0 & 0 & 0
\end{array}\right)\left(\begin{array}{c}
x^{2} \\
x \\
1
\end{array}\right)
\end{aligned}
$$




\section{F. Y8}

Introducing a dependent variable transformation $x_{n}=i y_{n}, a_{1}^{\prime}=i a_{1}$ to Eq. (Y

$$
x_{n+2} x_{n-1}=\frac{a_{0}+a_{1} x_{n}}{-a_{1} x_{n}+a_{0} x_{n} x_{n+1}}
$$

we obtain

$$
y_{n+2} y_{n-1}=\frac{a_{0}+a_{1}^{\prime} y_{n}}{a_{1}^{\prime} y_{n}+a_{0} y_{n} y_{n+1}} .
$$

This is equivalent to Eq. (Y7).

\section{G. Y9}

Introducing a variable transformation $x_{n}=\frac{\tilde{f}_{n+1}}{\widetilde{f}_{n}}$ to Eq. (Y9)

$$
x_{n+2} x_{n-1}=\frac{x_{n}+x_{n} x_{n+1}}{1+x_{n}}
$$

we obtain

$$
\frac{\widetilde{f}_{n+3} \widetilde{f}_{n}}{\widetilde{f}_{n+2}+\widetilde{f}_{n+1}}=\frac{\widetilde{f}_{n+2} \widetilde{f}_{n-1}}{\widetilde{f}_{n+1}+\widetilde{f}_{n}}
$$

From this equation, we obtain

$$
\widetilde{f}_{n+2} \widetilde{f}_{n-1}=\alpha\left(\widetilde{f}_{n+1}+\widetilde{f}_{n}\right)
$$

By scaling as $\tilde{f}_{n}=\alpha f_{n}$, we obtain

$$
f_{n+2} f_{n-1}=f_{n+1}+f_{n}
$$

This is Eq. (41) in the case of $a=0$.

\section{H. Y2}

Eq. (Y2) is written as

$$
x_{n+2} x_{n-1}=\frac{a+a x_{n}+a x_{n+1}+x_{n} x_{n+1}}{a+x_{n}+x_{n+1}+x_{n} x_{n+1}}
$$

where $a=a_{0} / a_{3}$. Eq. (Y2) is generated by a pair of second-order equation

$$
\left\{\begin{array}{l}
g_{n+1}=-g_{n}\left(1+\frac{\left(a+g_{n-1}\right)\left(b_{0}\left(a-g_{n}^{2}\right)-a c\right)}{(a-1)^{2}\left(a-g_{n}\right)\left(g_{n}+g_{n-1}\right)+b_{0} g_{n}\left(a+a g_{n}+a g_{n-1}+g_{n} g_{n-1}\right)-a c g_{n}}\right), \\
h_{n+1}=-h_{n}\left(1+\frac{\left(a+h_{n-1}\right)\left(b_{1}\left(a-h_{n}^{2}\right)-a c\right)}{(a-1)^{2}\left(a-h_{n}\right)\left(h_{n}+h_{n-1}\right)+b_{1} g_{n}\left(a+a h_{n}+a h_{n-1}+h_{n} h_{n-1}\right)-a c h_{n}}\right),
\end{array}\right.
$$


where $g_{n}=x_{2 n}, h_{n}=x_{2 n+1}$ and

$$
\begin{aligned}
c= & \frac{1}{x_{0} x_{1} x_{2}}\left(1+x_{0}\right)\left(1+x_{1}\right)\left(1+x_{2}\right)\left(a\left(1+x_{0}+x_{1}+x_{2}\right)+x_{0} x_{1}+x_{1} x_{2}+x_{2} x_{0}+x_{0} x_{1} x_{2}\right), \\
b_{0}= & \frac{1}{x_{0} x_{1} x_{2}}\left\{(a-1) x_{0} x_{2}\left(1+x_{1}\right)^{2}\right. \\
& \quad+\left(1+x_{1}+x_{0} x_{1}+x_{1} x_{2}\right)\left(a\left(1+x_{0}+x_{1}+x_{2}\right)+x_{0} x_{1}+x_{1} x_{2}+x_{2} x_{0}+x_{0} x_{1} x_{2}\right), \\
& \quad \\
b_{1}= & \quad \frac{1}{x_{1} x_{2} x_{3}}\left\{(a-1) x_{1} x_{3}\left(1+x_{2}\right)^{2}\right. \\
& \quad+\left(1+x_{2}+x_{1} x_{2}+x_{2} x_{3}\right)\left(a\left(1+x_{1}+x_{2}+x_{3}\right)+x_{1} x_{2}+x_{2} x_{3}+x_{3} x_{1}+x_{1} x_{2} x_{3}\right) .
\end{aligned}
$$

Eq. (51) does not belong to the QRT system as it stands, though there is still a possibility that it could be reduced to the system through a change of variable.

\section{Y3}

About Eq. (Y3), we can only show numerical results. For various parameter $a_{0}, a_{3}$ and initial values $x_{0} \sim x_{2}$, it is generated by a pair of second-order equations on even steps $\left(g_{n}\right)$ and odd steps $\left(h_{n}\right)$. In any case, both equations follows

$$
g_{n+1}=\frac{\sum_{0 \leq i, j \leq 3} a_{i j} g_{n-1}^{i} g_{n}^{j}}{\sum_{0 \leq i, j \leq 3} b_{i j} g_{n-1}^{i} g_{n}^{j}}, \quad h_{n+1}=\frac{\sum_{0 \leq i, j \leq 3} c_{i j} h_{n-1}^{i} h_{n}^{j}}{\sum_{0 \leq i, j \leq 3} d_{i j} h_{n-1}^{i} h_{n}^{j}},
$$

where $a_{i j} \sim d_{i j}$ are constant obtained from parameters and initial values numerically . This fact strongly suggests that Eq. (Y3) is also derived from a pair of second-order equations.

\section{ULTRADISCRETIZATION}

In this section, we consider an ultradiscrete version of the third-order integrable equations 13, 14]. Since ultradiscretization requires a positivity of parameters and dependent variables of the equations, (Y1),(Y2),(Y4),(Y6),(Y7) and (Y9) are ultradiscretizable. We show the procedure of ultradiscretization works well by Eq. (4) as an example. 
If we use transformations $y_{n}=\exp \left(\frac{Y_{n}}{\epsilon}\right), a=\exp \left(\frac{A}{\epsilon}\right)$ for Eq. (4)

$$
y_{n+2} y_{n-1}=a+y_{n}+y_{n+1}
$$

and take a limit $\epsilon \rightarrow+0$, then we have

$$
Y_{n+2}=\max \left(A, Y_{n}, Y_{n+1}\right)-Y_{n-1}
$$

This is an ultradiscrete version of Eq. (4). Fig 2 shows a trajectory of a solution to Eq. (56) in $3 \mathrm{D}$ phase space.

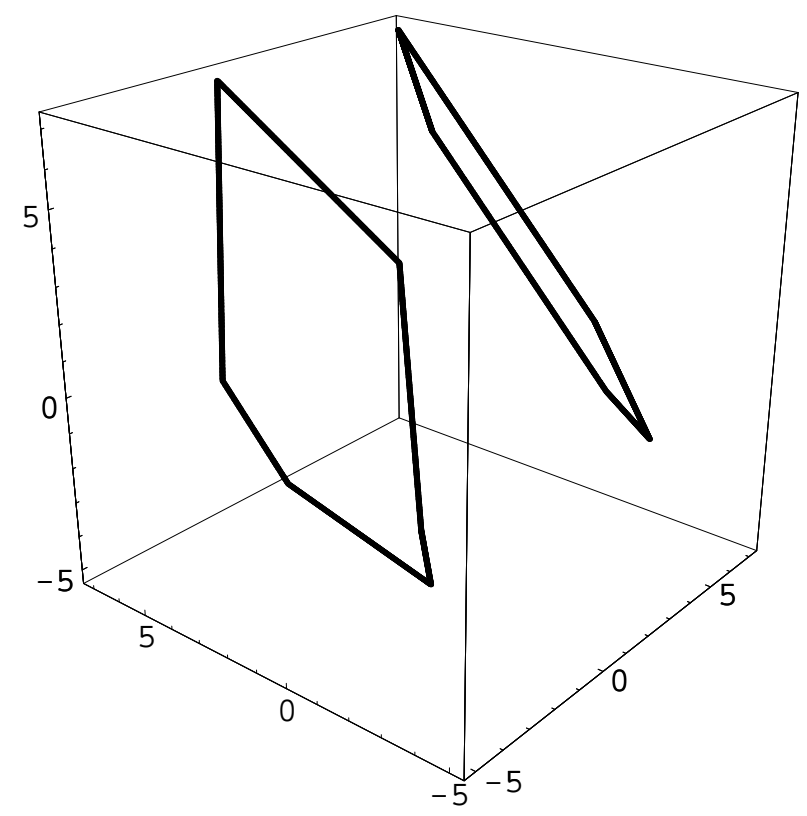

FIG. 2: A trajectory of a solution to Eq. (56) for $A=2.0, Y_{0}=2.0, Y_{1}=2.01, Y_{2}=-5.1$

It follows from the result for Eq. (44) in the previous section that Eq. (56) is generated by a pair of ultradiscrete QRT system

$$
\begin{aligned}
U_{n+1} & =\max \left(0, A+C_{0}, U_{n}+\max \left(0, C_{0}\right)\right)-U_{n-1}-\max \left(0, U_{n}\right), \\
V_{n+1} & =\max \left(0, A+C_{1}, V_{n}+\max \left(0, C_{1}\right)\right)-V_{n-1}-\max \left(0, V_{n}\right), \\
C_{0} & =\max \left(0, Y_{0}\right)+\max \left(0, Y_{2}\right)-Y_{1}, \\
C_{1} & =\max \left(0, Y_{1}\right)+\max \left(0, Y_{3}\right)-Y_{2},
\end{aligned}
$$


and invariant curves become

$$
\begin{aligned}
\max & \left(U_{n}+U_{n+1}, \max \left(U_{n}, U_{n+1}\right)+\max \left(0, C_{0}\right), \max \left(U_{n}-U_{n+1}, U_{n+1}-U_{n}\right)+\max \left(0, C_{0}\right),\right. \\
& \max \left(-U_{n},-U_{n+1}\right)+\max \left(0, C_{0}, A+C_{0}, 2 C_{0}\right), \\
& \left.-U_{n}-U_{n+1}+\max \left(0, C_{0}\right)+\max \left(0, A+C_{0}\right)\right)=K_{0}
\end{aligned}
$$

and

$$
\begin{aligned}
\max & \left(V_{n}+V_{n+1}, \max \left(V_{n}, V_{n+1}\right)+\max \left(0, C_{1}\right), \max \left(V_{n}-V_{n+1}, V_{n+1}-V_{n}\right)+\max \left(0, C_{1}\right),\right. \\
& \max \left(-V_{n},-V_{n+1}\right)+\max \left(0, C_{1}, A+C_{1}, 2 C_{1}\right), \\
& \left.-V_{n}-V_{n+1}+\max \left(0, C_{1}\right)+\max \left(0, A+C_{1}\right)\right)=K_{1}
\end{aligned}
$$

Fig 3 shows invariant curves for Eq. (157) determined by Eq. (61).

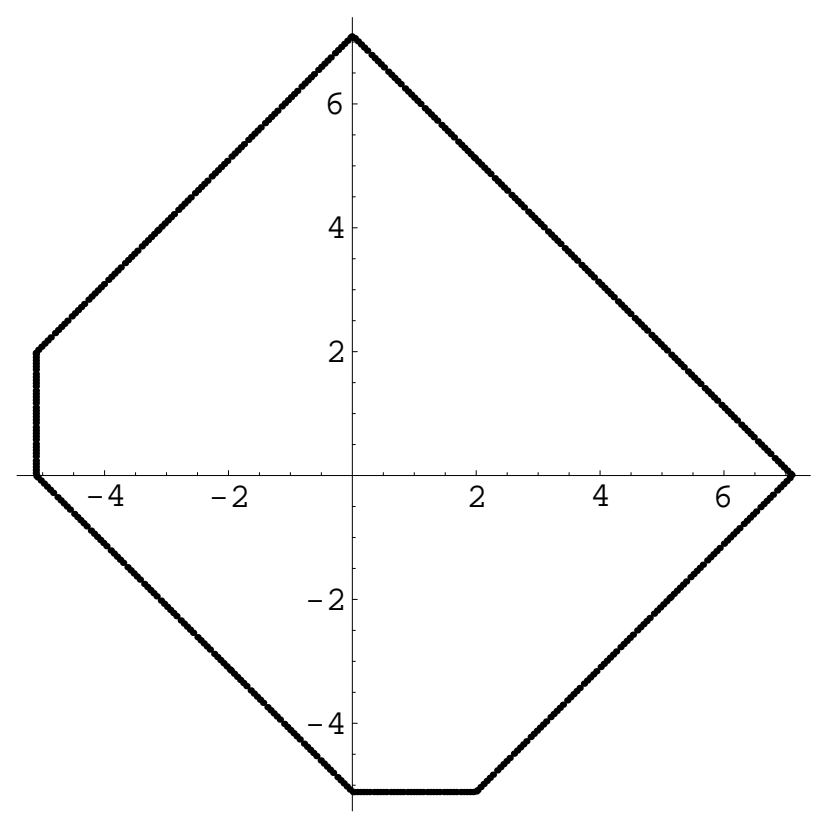

FIG. 3: An invariant curve of Eq. (57) for $A=2.0, Y_{0}=2.0, Y_{1}=2.01, Y_{2}=-5.1$

\section{CONCLUSION}

In this paper, we have shown the third-order integrable difference equations proposed by Hirota, Kimura and Yahagi are generated by a pair of second-order integrable difference equations. In the case of Eqs. (1Y1) and (1Y4) ( (Y9), second-order difference equations 
are a special case of the QRT system. In the case of Eqs. (Y2) and (Y3), second-order equations may not be the QRT system. Furthermore we have shown the procedure of ultradiscretization works well for third-order equation, and derived second-order equations and invariants curves are also ultradiscretizable .

Although the whole integrability structure of the general third-order equations is still unknown, our work would be one of keys to understand the structure. Generating our results, that is, investigating a connection between the general QRT system and the thirdorder equations is an important future problem.

\section{Acknowledgments}

The authors express our sincere thanks to Professor Ryogo Hirota for fruitful discussions and encouragement.

[1] B. Grammaticos, A. Ramani, and V. Papageorgiou, Phys. Rev. Lett. 67, 1825 (1991).

[2] J. Hietarinta and C. Viallet, Phys. Rev. Lett. 81, 325 (1998).

[3] H. Sakai, Commun. Math. Phys. 220, 165 (2001).

[4] T. Takenawa, J. Phys. A. 34, L95 (2001).

[5] A. Ramani, B. Grammaticos, and Y. Ohta, Commun. Math. Phys. 217, 315 (2001).

[6] A. Ramani, B. Grammaticos, and Y. Ohta, J. Phys. A. 34, 2505 (2001).

[7] T. Tsuda, J. Phys. A. 37, 2721 (2004).

[8] K. Kajiwara, T. Masuda, M. Noumi, Y. Ohta, and Y. Yamada, J. Phys. A. 36, L263 (2003).

[9] G. Quispel, J. Robert, and C. Thompson, Phys. Lett. A. 126, 419 (1988).

[10] G. Quispel, J. Robert, and C. Thompson, Physica. D 34, 183 (1989).

[11] A. Ramani, B. Grammaticos, and J. Hietarinta, Phys. Rev. Lett. 67, 1829 (1991).

[12] R. Hirota, K. Kimura, and H. Yahagi, J. Phys. A. 34, 10377 (2001).

[13] T. Tokihiro, D. Takahashi, J. Matsukidaira, and J. Satsuma, Phys. Rev. Lett. 76, 3247 (1996).

[14] J. Matsukidaira, J. Satsuma, D. Takahashi, T. Tokihiro, and M. Torii, Phys. Lett. A. 255, 287 (1997). 